\title{
HQET sum rules for quark-gluon three-body components in the $B$ meson
}

\section{Tetsuo Nishikawa}

Faculty of Health Science, Ryotokuji University, Urayasu, Chiba 279-8567, Japan

E-mail: nishikawa@ryotokuji-u.ac.jp

\section{Kazuhiro Tanaka*}

Department of Physics, Juntendo University, Inzai, Chiba 270-1695, Japan

E-mail: tanakakesakura.juntendo.ac.jp

\begin{abstract}
We discuss the heavy-quark effective theory (HQET) parameters, $\lambda_{E}$ and $\lambda_{H}$, which correspond to matrix elements representing quark-gluon three-body components in the $B$-meson wavefunction. We derive the QCD sum rules for $\lambda_{E, H}$ calculating the new higher-order contributions to the operator product expansion for the relevant correlator in the HQET, i.e., the order $\alpha_{s}$ radiative corrections to the Wilson coefficients associated with the dimension-5 quark-gluon mixed operator, and the power corrections due to the dimension- 6 four-quark operators. We find that the new radiative corrections significantly improve the stability of the corresponding Borel sum rules and lead to the reduction of the values of $\lambda_{E, H}$. We also discuss the improvement for the sum rules with the resummation of the relevant logarithms of the $b$-quark mass based on the renormalization group and present update on the values of $\lambda_{E, H}$.
\end{abstract}

Sixth International Conference on Quarks and Nuclear Physics

April 16-20, 2012

Ecole Polytechnique, Palaiseau, Paris

\footnotetext{
*Speaker.
} 


\section{Introduction}

The decays of the $B$ mesons play distinguished roles in exploring $\mathrm{CP}$ violation and the flavor sector of the Standard Model [1]. In the heavy-quark limit based on $\Lambda_{\mathrm{QCD}} / m_{b} \ll 1$ with $m_{b}$ being the $b$-quark mass, the $B$-meson matrix elements relevant for those decays obey heavy-quark symmetry, and are described by the heavy-quark effective theory (HQET) [2]. Here, fundamental quantities are the HQET parameters that are defined as matrix elements of the relevant local operators, like the decay constant [2]: $\left\langle 0\left|\bar{q} \gamma_{\rho} \gamma_{5} h_{v}\right| \bar{B}(v)\right\rangle=i F(\mu) v_{\rho}$, where $|\bar{B}(v)\rangle$ is the $\bar{B}$-meson state with the 4-velocity $v$ in the HQET, $\bar{q}$ is the light-antiquark field, $h_{v}$ is the effective heavy-quark field as $b(x)=e^{-i m_{b} v \cdot x} h_{v}(x)+O\left(1 / m_{b}\right)$, with $v h_{v}(x)=h_{v}(x)$, and the heavy-light local operator in the LHS is renormalized at the scale $\mu$. The decay constant $F(\mu)$ represents the magnitude of the valence Fock content in the $B$-meson wavefunction in the heavy-quark limit, and thus determines the normalization of the amplitude for exclusive $B$-meson decays. The heavy-quark expansion gives, $f_{B} \sqrt{m_{B}}=F(\mu)\left[1+\left(C_{F} \alpha_{s} / 4 \pi\right)\left(3 \ln \left(m_{b} / \mu\right)-2\right)+\ldots\right]+O\left(1 / m_{b}\right)$, and relates $F(\mu)$ to the physical decay constant $f_{B}$ [2], whose value is now obtained rather precisely from lattice QCD calculations.

We can also define the analogues of $F(\mu)$, which are associated with the higher Fock components inside the $B$ meson. For the non-minimal parton configurations with additional gluons, the corresponding HQET parameters are defined as [3]

$$
\left\langle 0\left|\bar{q} \boldsymbol{\alpha} \cdot g \boldsymbol{E} \gamma_{5} h_{v}\right| \bar{B}(v)\right\rangle=F(\mu) \lambda_{E}^{2}(\mu), \quad\left\langle 0\left|\bar{q} \boldsymbol{\sigma} \cdot g \boldsymbol{H} \gamma_{5} h_{v}\right| \bar{B}(v)\right\rangle=i F(\mu) \lambda_{H}^{2}(\mu),
$$

in terms of the matrix elements in the $B$-meson rest frame with $v=(1, \mathbf{0})$. Here, the three-body quark-gluon operators are associated with the chromoelectric and chromomagnetic fields, $E^{i}=G^{0 i}$ and $H^{i}=(-1 / 2) \varepsilon^{i j k} G^{j k}$, with $G_{\mu \nu}$ being the gluon field strength tensor. The values of $\lambda_{E, H}^{2}$ were estimated in [3] using QCD sum rules, as

$$
\lambda_{E}^{2}(\mu)=0.11 \pm 0.06 \mathrm{GeV}^{2}, \quad \lambda_{H}^{2}(\mu)=0.18 \pm 0.07 \mathrm{GeV}^{2},
$$

at $\mu=1 \mathrm{GeV}$; besides this rather rough estimate, there has been no other estimate. Recently, it has been demonstrated that the $B$-meson valence Fock light-cone distribution amplitude $\phi_{+}(\omega, \mu)[3,4]$

$$
\frac{1}{i F(\mu)}\left\langle 0\left|\bar{q}(t n) \mathscr{P} \exp \left[i g \int_{0}^{t} d \lambda n \cdot A(\lambda n)\right] h \gamma_{5} h_{v}(0)\right| \bar{B}(v)\right\rangle=\int d \omega e^{-i \omega t} \phi_{+}(\omega, \mu),
$$

with $n_{\mu}$ as the light-like vector $\left(n^{2}=0, n \cdot v=1\right)$, is significantly contaminated by the multiparticle Fock states [5], so that the contributions represented by the novel HQET parameters $\lambda_{E, H}^{2}$ of (1.1) could strongly affect the amplitudes for the exclusive $B$-meson decays [6]. Therefore, an improved estimate of $\lambda_{E, H}^{2}$ is expected to give a better control of the hadronic uncertainty which is a major source of theoretical uncertainty in the calculations of the $B$-meson decay rates [1]. In this report, we update the QCD sum rule calculation of $\lambda_{E, H}^{2}$ with the higher-order contributions in the HQET.

\section{QCD sum rules in the HQET}

We consider the correlator between the two- and three-body currents in the HQET,

$$
\begin{aligned}
& i \int d^{4} x e^{-i \omega v \cdot x}\left\langle 0\left|T\left[\bar{q}(0) \Gamma_{1} g G_{\mu v}(0) h_{v}(0) \bar{h}_{v}(x) \Gamma_{2} q(x)\right]\right| 0\right\rangle \\
& \quad=-\frac{1}{2} \operatorname{Tr}\left[\sigma_{\mu v} \Gamma_{1} P_{+} \Gamma_{2}\right] \Pi_{3 H}(\omega)-\frac{1}{2} \operatorname{Tr}\left[\left(i v_{\mu} \gamma_{v}-i v_{v} \gamma_{\mu}\right) \Gamma_{1} P_{+} \Gamma_{2}\right] \Pi_{3 S}(\omega),
\end{aligned}
$$




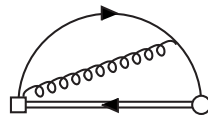

(a)

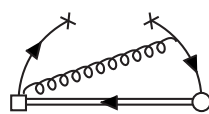

(b)

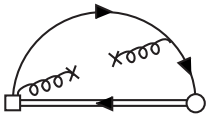

(c)

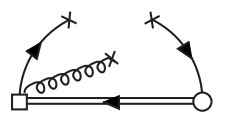

(d)

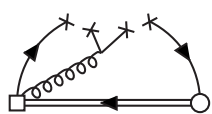

(e)

Figure 1: Nonvanishing diagrams for the OPE of the correlator (2.1) in the Fock-Schwinger gauge. The double line denotes the propagator of the heavy quark, and the circle and box represent the relevant currents.

where $P_{+}=(1+\boldsymbol{v}) / 2$ is the projector on the upper components of heavy-quark spinor, $\Gamma_{1}$ is an arbitrary gamma matrix, and we choose $\Gamma_{2}=\gamma_{5}$ to construct sum rules for pseudoscalar $B$ meson. $\Pi_{3 H}(\omega)$ and $\Pi_{3 S}(\omega)$ provide sum rules to evaluate $\lambda_{H}^{2}$ and the "splitting" $\lambda_{H}^{2}-\lambda_{E}^{2}$, respectively, based on the corresponding dispersion relations,

$$
\Pi_{3 H}(\omega)=\frac{F^{2}(\mu) \lambda_{H}^{2}(\mu)}{6(\bar{\Lambda}-\omega-i 0)}+\cdots, \quad \Pi_{3 S}(\omega)=\frac{F^{2}(\mu)\left[\lambda_{H}^{2}(\mu)-\lambda_{E}^{2}(\mu)\right]}{6(\bar{\Lambda}-\omega-i 0)}+\cdots
$$

with a pole at the "effective mass" $\bar{\Lambda}\left(=m_{B}-m_{b}\right)$ of the $B$-meson in the HQET and the ellipses standing for the pole contributions of higher resonances and the continuum contributions. We note that, with the formal substitution $g G_{\mu v}(0) \rightarrow 1$ in the LHS in $(2.1)$, the RHS reduces to $(-1 / 2) \operatorname{Tr}\left[\Gamma_{1} P_{+} \Gamma_{2}\right] \Pi_{F}(\omega)$, where the corresponding correlation function obeys the dispersion relation, $\Pi_{F}(\omega)=F^{2}(\mu) /[2(\bar{\Lambda}-\omega-i 0)]+\cdots$, and thus provides a sum rule to evaluate $F(\mu)[2,7,8]$.

The matching of the dispersion relations of $(2.2)$ with $(X=3 H, 3 S)$

$$
\left.\Pi_{X}(\omega)\right|_{-\omega \gg \Lambda_{\mathrm{QCD}}}=C_{I}^{X}(\omega)+C_{q}^{X}(\omega)\langle\bar{q} q\rangle+C_{G}^{X}(\omega)\left\langle G^{2}\right\rangle+C_{\sigma}^{X}(\omega)\langle\bar{q} g G \cdot \sigma q\rangle+\cdots,
$$

which are obtained by the operator product expansion (OPE) of the correlator (2.1) in the region $-\omega \gg \Lambda_{\mathrm{QCD}}$, yields the corresponding sum rules. Here, the usual vacuum condensates, $\langle\bar{q} q\rangle \equiv\langle 0|\bar{q} q| 0\rangle,\left\langle G^{2}\right\rangle \equiv\left\langle 0\left|\left(G_{\mu \nu}^{a}\right)^{2}\right| 0\right\rangle,\langle g G \cdot \sigma q\rangle \equiv\left\langle 0\left|\bar{q} g G^{\mu v} \sigma_{\mu \nu} q\right| 0\right\rangle, \ldots$, arise with increasing dimension, which implies extra powers of $1 / \omega$ for the corresponding Wilson coefficients $C_{k}^{X}(\omega)$. The OPE (2.3) at the leading accuracy in $\alpha_{s}$ is represented by the diagrams in Fig. 1, here, we use the Fock-Schwinger gauge, $x^{\mu} A_{\mu}(x)=0$, for the background gluon field, so that the heavy quark does not interact with the nonperturbative gluons in the calculation for power corrections. The diagrams (a)-(d) generate, respectively, the first four terms of (2.3), as obtained in [3]. The matching between (2.3) and (2.2) is performed for their Borel transforms, $B_{M}\left[\Pi_{X}(\omega)\right]=$ $\int_{0}^{\infty}(d \omega / \pi M) e^{-\omega / M} \operatorname{Im} \Pi_{X}(\omega)$, to suppress the power-correction terms in (2.3) and the contributions of higher resonances and continuum in (2.2). This allows us to obtain the usual Borel sum rules as functions of the Borel parameter $M$, as well as the "continuum threshold" $\omega_{\text {th }}$ above which the the spectral functions relevant to (2.2) are approximated by those based on the OPE (2.3). Using the standard values of the condensates and eliminating $F^{2}(\mu)$ in favor of the similar Borel sum rules derived from $\Pi_{F}(\omega)$, we obtain the sum rules for $\lambda_{E, H}^{2}$, which depend sizeably on the parameter $M$ and thus yield the above-mentioned estimate (1.2) with rather large errors (see Fig. 3 below).

\section{Higher order corrections in the OPE: power corrections and radiative corrections}

Now, we consider the QCD corrections beyond the above results. We first calculate the leading correction term arising in the ellipses of (2.3), i.e., the power correction due to the diagram (e) in 


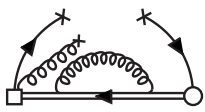

(a)

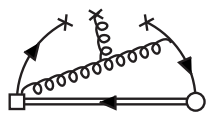

(g)

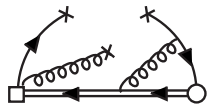

(b)

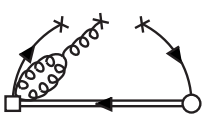

(h)
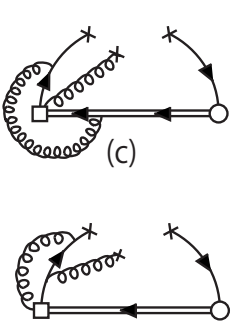

(i)

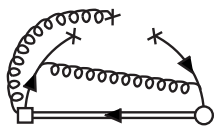

(d)

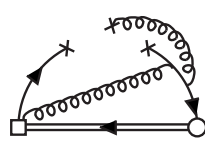

(j)

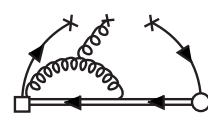

(e)

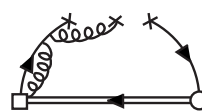

(k)

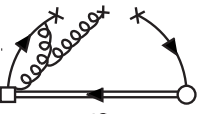

$(\mathrm{f})$

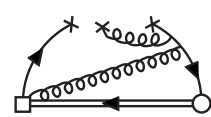

(I)

Figure 2: Diagrams of the correlator (2.1) in the Fock-Schwinger gauge, relevant for the one-loop matching of the Wilson coefficients associated with the dimension-5 quark-gluon-mixed condensate in (2.3).

Fig. 1, induced by the dimension-6 four-quark condensates $\sim\left\langle\left(\bar{q} \gamma_{\mu} T^{a} q\right)^{2}\right\rangle[11]$. We find that the corresponding corrections to the Borel sum rules for $\lambda_{E, H}^{2}$ are smaller than the dominant effects from the lower-dimensional condensates, such that the convergence of the OPE (2.3) is suggested at this level of power corrections.

Next, we calculate the new order $\alpha_{s}$ corrections to the sum rules for $\lambda_{E, H}^{2}$. Fig. 1 shows that, among the Wilson coefficients of (2.3) used in the above-mentioned estimate (1.2) in [3], only $C_{\sigma}^{X}(\omega)$, associated with the dimension-5 quark-gluon-mixed condensate $\langle\bar{q} g G \cdot \sigma q\rangle$, was calculated at the level of $O\left(\alpha_{s}^{0}\right)$, while all the others were of $O\left(\alpha_{s}\right)$. The one-loop diagrams in Fig. 2 for the correlator (2.1) are relevant to the matching at $O\left(\alpha_{s}\right)$ for $C_{\sigma}^{X}(\omega)$. We calculate those diagrams in $D=4+2 \varepsilon$ dimensions and derive the $O\left(\alpha_{s}\right)$ correction to $C_{\sigma}^{X}(\omega)$ in the $\overline{\mathrm{MS}}$ scheme: the diagrams (a)-(c) and (e) are all UV-divergent, while the diagrams (g), (j) and (l) are IR-divergent. Each of the diagrams (f), (h), (i) and (k) vanishes as a result of the "canceling" UV and IR poles, $1 / \varepsilon_{U V}-1 / \varepsilon_{I R}$, arising from the scaleless loop integral. On the other hand, the diagram (d) is of $O(\varepsilon)$. All those UV poles are subtracted according to the $\overline{\mathrm{MS}}$ renormalization of the fields and currents relevant to the correlator (2.1), in particular, taking into account the renormalization mixing of the (many) quark-gluon three-body currents, while the sum of the IR poles is absorbed into the matrix element $\langle\bar{q} g G \cdot \sigma q\rangle$ with the renormalized dimension-5 operator. The remaining finite contribution determines $C_{\sigma}^{X}(\omega)$ at $O\left(\alpha_{s}\right)$; the corresponding NLO Wilson coefficients read [11]

$$
\begin{aligned}
C_{\sigma}^{3 H}(\omega) & =\frac{1}{24 \omega}\left[1-\frac{\alpha_{s}}{4 \pi}\left\{\left(N_{c}-\frac{6}{N_{c}}\right) \ln \frac{-2 \omega}{\mu}-\frac{5}{2} N_{c}+\frac{1}{N_{c}}\right\}\right], \\
C_{\sigma}^{3 S}(\omega) & =\frac{\alpha_{s}}{96 \pi \omega}\left[N_{c} \ln \frac{-2 \omega}{\mu}+\frac{1}{2 N_{c}}\right],
\end{aligned}
$$

in the $\overline{\mathrm{MS}}$ scheme. Combined with the above results, (3.1) and (3.2) allow us to obtain the Borel sum rules for $\lambda_{E, H}^{2}(\mu)$, with the perturbative corrections up to $O\left(\alpha_{s}\right)$ accuracy, as well as with the power corrections up to those due to the dimension- 6 condensates.

\section{Renormalization-group improvement and Borel analysis}

The new $O\left(\alpha_{s}\right)$ contributions due to Fig. 2 bring an explicit dependence on the scale $\mu$ through the logarithmic term in (3.1) and (3.2), which produces the term proportional to $\ln (M / \mu)$ in the corresponding Borel sum rules; a remarkable point is that this new $\mu$ dependence, combined with that 

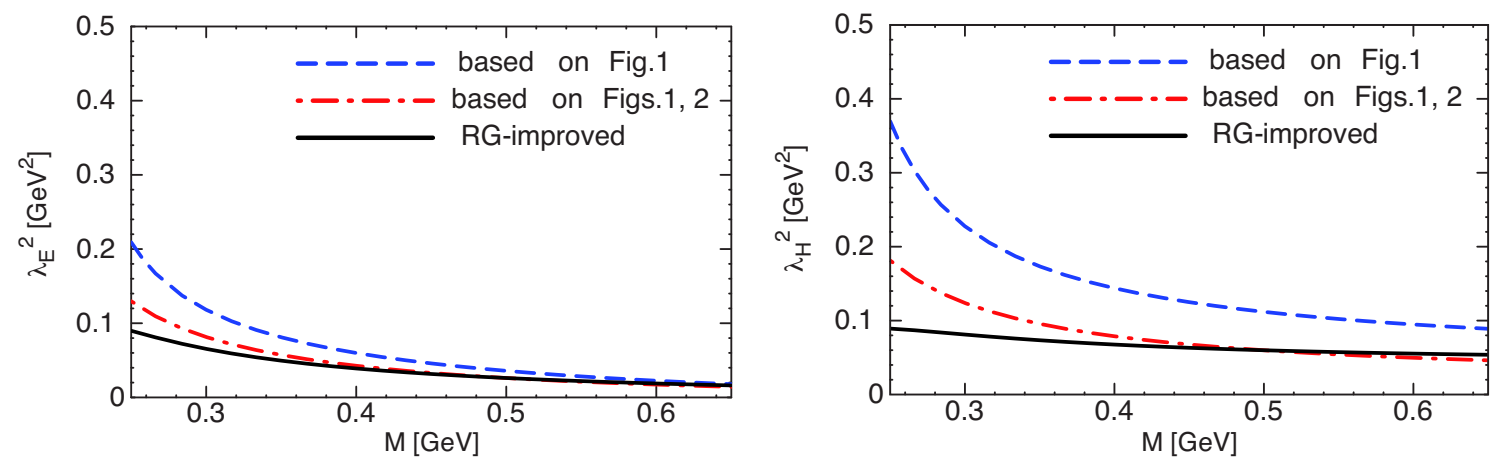

Figure 3: The Borel sum rules for $\lambda_{E, H}^{2}$ at $\mu=1 \mathrm{GeV}$ with the continuum threshold $\omega_{\mathrm{th}}=1.3 \mathrm{GeV}$.

of the relevant condensates, makes our sum-rule formulae for $\lambda_{E, H}^{2}(\mu)$ obey the renormalization group (RG) equations with the correct one-loop mixing matrix [9, 6] between $\lambda_{E}^{2}(\mu)$ and $\lambda_{H}^{2}(\mu)$. This fact allows us to perform further the RG improvement of our sum-rule formulae for $\lambda_{E, H}^{2}(\mu)$, such that the effects associated with $\alpha_{s} \ln (M / \mu)$ are resummed to all orders by taking into account the anomalous dimensions of the relevant condensates. We eventually obtain $\lambda_{E, H}^{2}(\mu=1 \mathrm{GeV})$ based on the RG evolutions for $\lambda_{E, H}^{2}(\mu)$.

In Fig. 3 we show the results for $\lambda_{E, H}^{2}(\mu=1 \mathrm{GeV})$ as functions of the Borel parameter $M$ using our Borel-transformed sum-rule formulae [11] with the continuum threshold $\omega_{\mathrm{th}}=1.3 \mathrm{GeV}$ : the OPE represented by Fig. 1 yields the dashed curve, whose behaviors are close to the results of [3], and the OPE represented by Figs. 1 and 2 yields the dot-dashed curve. The RG improvement of the latter case yields the solid curve as our full result. The new $O\left(\alpha_{s}\right)$ contributions due to Fig. 2, and also the associated RG effects, significantly improve the stability of the sum rules. Furthermore, those contributions significantly reduce the magnitude of $\lambda_{H}^{2}$ as well as $\lambda_{E}^{2}$. We find the similar stability of the RG-improved results in the "window" $0.4 \mathrm{GeV} \lesssim M \lesssim 0.6 \mathrm{GeV}$, for the cases with $\omega_{\mathrm{th}}=1.2-1.4 \mathrm{GeV}$. These results lead to an update on the values of $\lambda_{E, H}^{2}$ as [11]

$$
\lambda_{E}^{2}(1 \mathrm{GeV})=0.03 \pm 0.02 \mathrm{GeV}^{2}, \quad \lambda_{H}^{2}(1 \mathrm{GeV})=0.06 \pm 0.03 \mathrm{GeV}^{2},
$$

where the errors take into account the uncertainties due to the choice of the continuum threshold $\omega_{\mathrm{th}}$ and the dependence on the Borel parameter $M$, and also the other sources of uncertainties, i.e., uncertainties in the empirical input parameters, the vacuum condensates and $\Lambda_{\mathrm{QCD}}$, and uncertainties due to the lack of information of the higher-loop effects in the OPE. Compared with (1.2), the central values of our result (4.1) are smaller by $1 / 3$ and the errors are also reduced considerably. We note that, in the sum rules for the decay constant $F(\mu)$, the large effect from the order- $\alpha_{s}$ radiative corrections, mainly due to Coulomb interaction between heavy and light quarks, was observed to enhance $F(1 \mathrm{GeV})$ significantly [7, 2]. This effect actually plays important roles, through (2.2), in the reduction of (4.1) compared with (1.2). We mention that the sum rules for $F(\mu)$ are quite stable with respect to inclusion of the order- $\alpha_{s}^{2}$ radiative corrections [8].

\section{Conclusions}

We have updated the sum rules for the HQET parameters $\lambda_{E, H}^{2}$ calculating the new higher-order 
contributions to the OPE for the corresponding HQET correlator. We have found that the new order- $\alpha_{s}$ radiative corrections to the Wilson coefficients associated with the dimension-5 quarkgluon mixed condensate significantly reduce the values of $\lambda_{E, H}^{2}$, and also make the corresponding sum rule formulas for $\lambda_{E, H}^{2}$ obey the correct RG equations. Our final result is obtained as (4.1), where the perturbative as well as nonperturbative corrections are under control and the various sources of errors are taken into account.

One might anticipate that the higher Fock components (1.1) in the $B$-meson wavefunction would be associated with the "higher-twist" power corrections to the exclusive $B$-decay amplitudes, as in the wavefunctions for the light mesons $\pi, \rho$, etc. However, the presence of a heavy quark inside the $B$ meson causes nonperturbative quark-gluon interactions which induce the mixing of (1.1) to the leading-twist distribution amplitude (1.3) [3, 5, 6], and the integral, $\lambda_{B}^{-1}(\mu) \equiv$ $\int_{0}^{\infty} d \omega \phi_{+}(\omega, \mu) / \omega$, determines the normalization of the so-called hard spectator interaction amplitude [1] that contributes to the exclusive $B$-decay amplitudes at the leading power [6]: using the previous estimate (1.2), $\lambda_{B}^{-1}(1 \mathrm{GeV}) \simeq 2.7 \mathrm{GeV}^{-1}$ was obtained as a result of the most sophisticated QCD calculation [6] and was larger than various theoretical estimates of $\lambda_{B}^{-1}[1,4,10]$. Now, using our new result (4.1), we find that $\lambda_{B}^{-1}$ becomes close to the other estimates as $\lambda_{B}^{-1}(1 \mathrm{GeV})=$ $2.0 \pm 0.2 \mathrm{GeV}^{-1}$, with the error being due to the uncertainty in (4.1) only. Investigations of exclusive $B$-meson decays using the present results will be reported elsewhere [12].

\section{Acknowledgements}

This work was supported by the Grant-in-Aid for Scientific Research No. B-19340063. The work of K. T. was supported in part by the Grant-in-Aid for Scientific Research on Priority Areas No. 22011012 and the Grant-in-Aid for Scientific Research No. C-23540292.

\section{References}

[1] M. Antonelli et al., Phys. Rept. 494 (2010) 197.

[2] M. Neubert, Phys. Rept. 245 (1994) 259.

[3] A. G. Grozin and M. Neubert, Phys. Rev. D55 (1997) 272.

[4] V. M. Braun, D. Y. Ivanov and G. P. Korchemsky, Phys. Rev. D69 (2004) 034014; S. J. Lee and M. Neubert, Phys. Rev. D72 (2005) 094028.

[5] H. Kawamura, J. Kodaira, C.F. Qiao and K. Tanaka, Phys. Lett. B523 (2001) 111 [Erratum-ibid. B536 (2002) 344]; Mod. Phys. Lett. A18 (2003) 799.

[6] H. Kawamura and K. Tanaka, Phys. Lett. B673 (2009) 201; Phys. Rev. D81 (2010) 114009.

[7] E. Bagan, P. Ball, V. M. Braun and H. G. Dosch, Phys. Lett. B278 (1992) 457.

[8] A. A. Penin and M. Steinhauser, Phys. Rev. D65 (2002) 054006.

[9] A. G. Grozin and M. Neubert, Nucl. Phys. B495 (1997) 81.

[10] A. Khodjamirian, T. Mannel and N. Offen, Phys. Rev. D75 (2007) 054013.

[11] T. Nishikawa and K. Tanaka, arXiv:1109.6786 [hep-ph].

[12] Y. -C. Chen and H. -n. Li, Phys. Lett. B712 (2012) 63; H. Kawamura and K. Tanaka, in preparation. 